\title{
Generic Method to Create Segregated Structures toward Robust, Flexible, Highly Conductive Elastomer Composites
}

Chengfeng Zhang, Zhenghai Tang, * Xinglong An, Shifeng Fang, Siwu Wu and Baochun Guo*

Department of Polymer Materials and Engineering, South China University of Technology, Guangzhou

\author{
510640, P.R. China
}

*E-mail: mszhtang@ scut.edu.cn.

*E-mail: psbcguo@scut.edu.cn.

\section{Figure and Table}

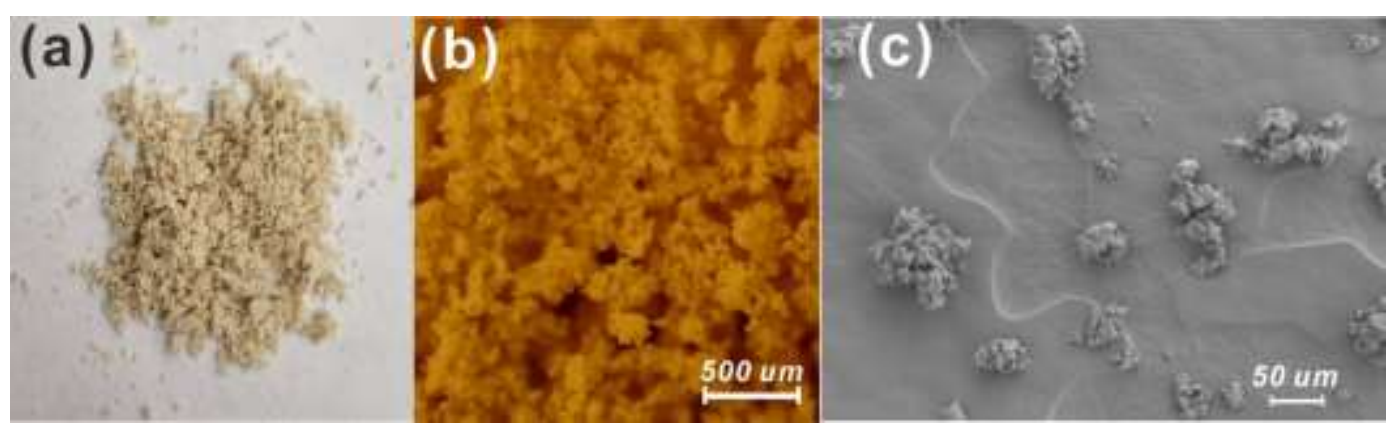

Figure S1. (a) Digital photograph, (b) optical microscopic image and (c) SEM image of NR-RGs. 
(a)

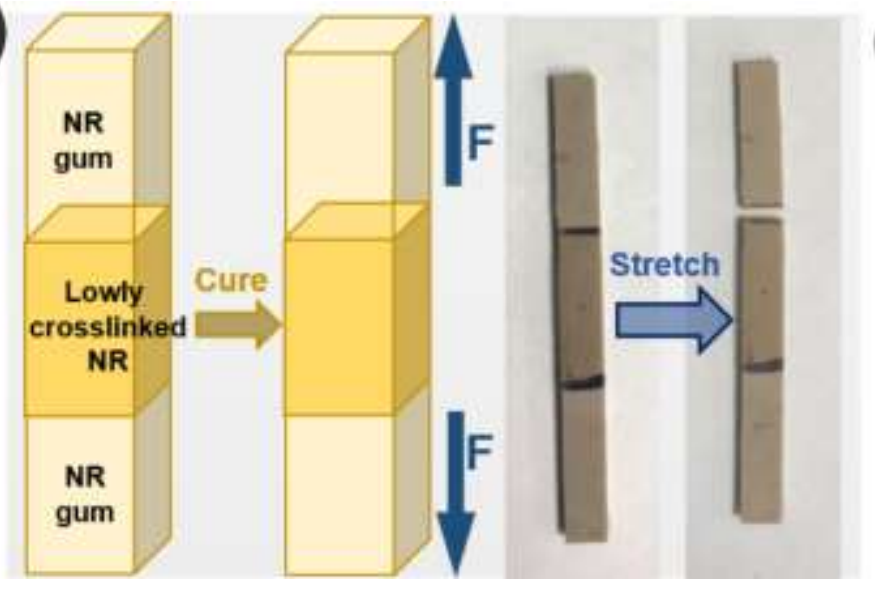

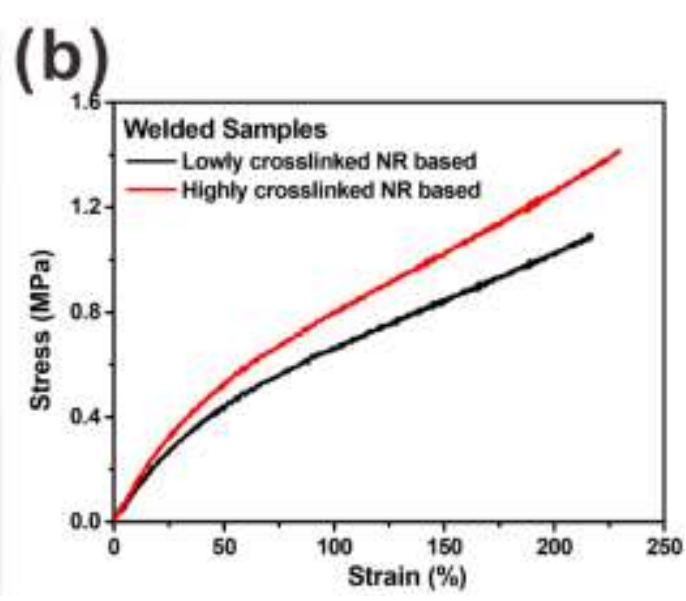

Figure S2. (a) Schematic and photographs of weld experiment by bringing two parts of uncured NR compounds and a cured NR strip (the same formulation as that for H-NR/CNTs-0) together following by pressing at $143^{\circ} \mathrm{C}$ for the optimum time. (b) Stress-strain curves of the welded samples.

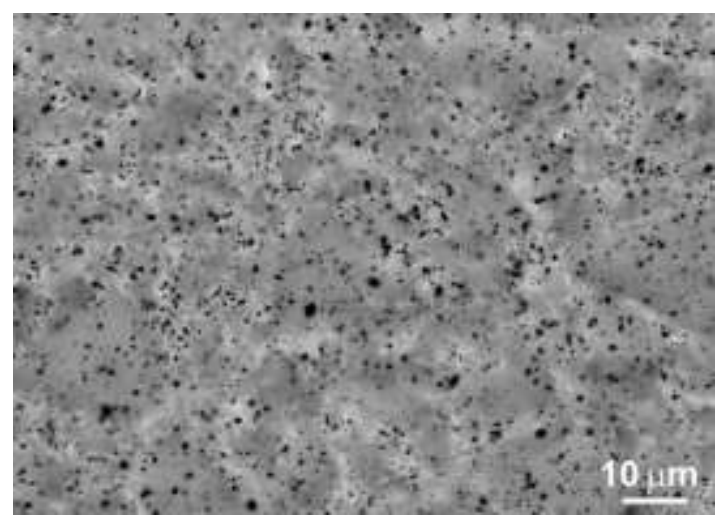

Figure S3. Field-emission SEM image of S-NR/CNTs-0. 


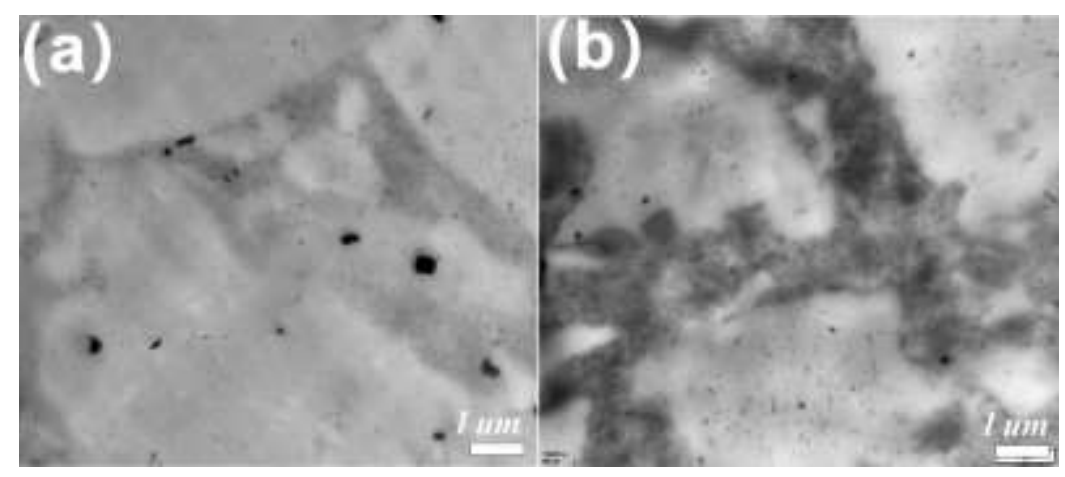

Figure S4. TEM images of (a) S-NR/CNTs-0.94 and (b) S-NR/CNTs-2.78.
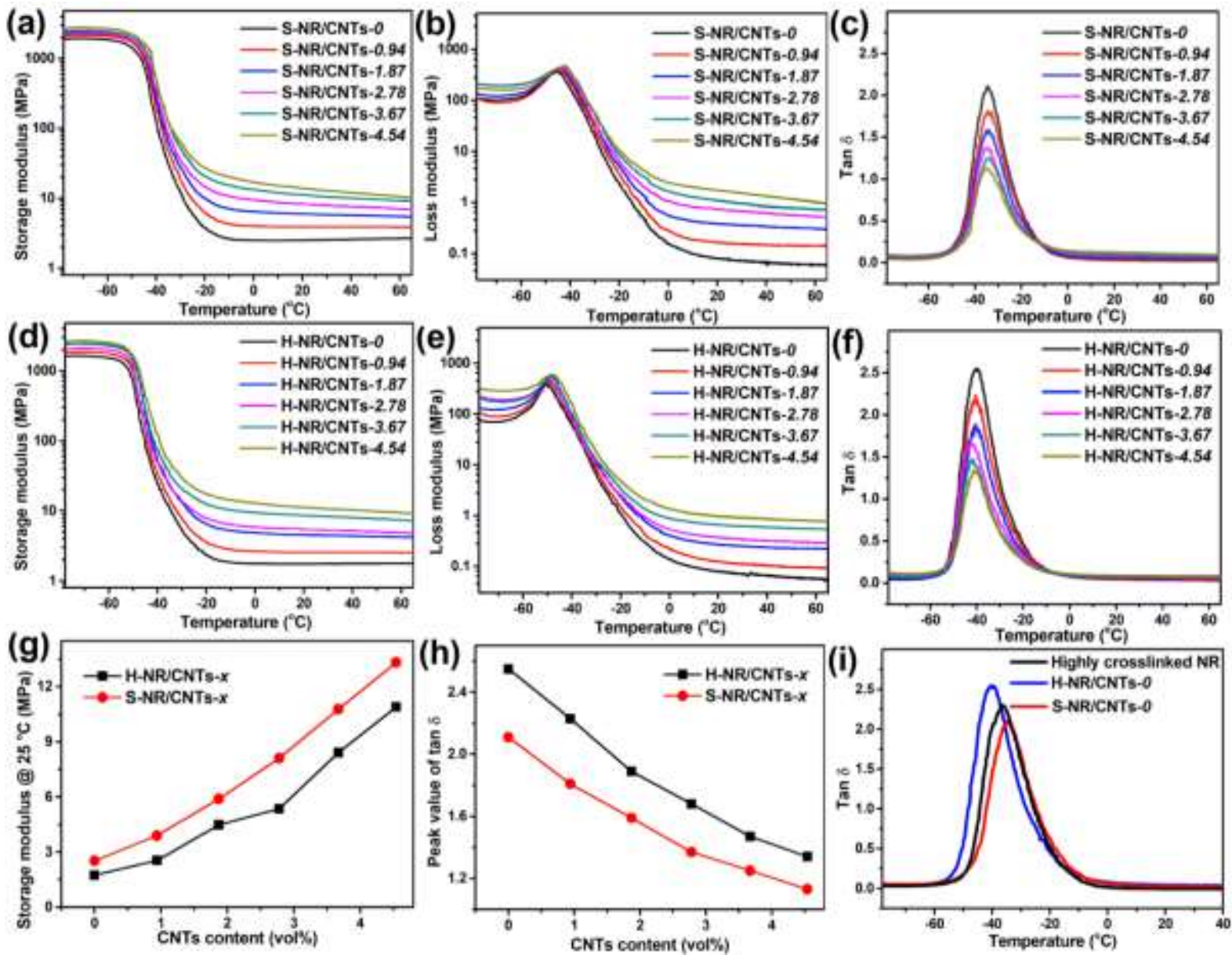

Figure S5. Dependence of (a) storage modulus, (b) loss modulus, and (c) $\tan \delta$ on temperature for S-NR/CNTs-

$x$. Dependence of (d) storage modulus, (e) loss modulus, and (f) $\tan \delta$ on temperature for H-NR/CNTs- $x$. (g)

Comparison on the storage modulus at $25{ }^{\circ} \mathrm{C}$ for S-NR/CNTs- $x$ and H-NR/CNTs- $x$. (h) Comparison on the tan S-3 
$\delta$ peak values for S-NR/CNTs- $x$ and H-NR/CNTs- $x$. (i) Comparison on the tan $\delta$ curves for S-NR/CNTs- 0, HNR/CNTs-0 and highly crosslinked NR.

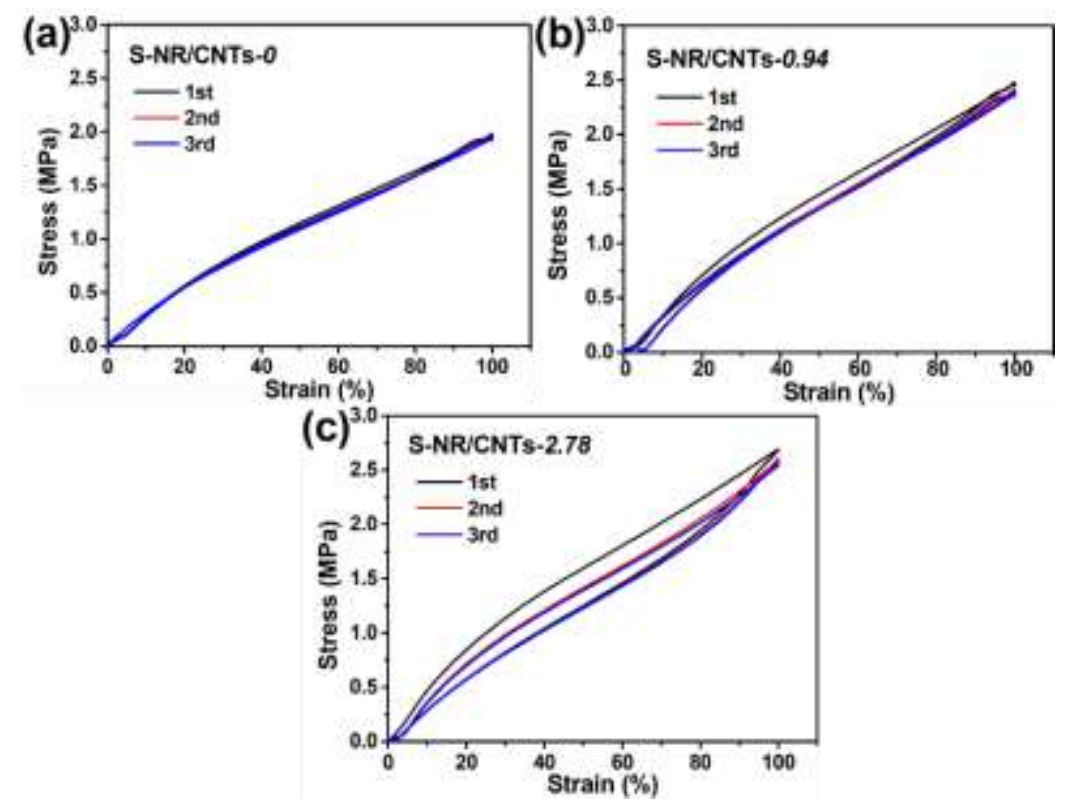

Figure S6. Cyclic tensile curves of (a) S-NR/CNTs-0, (b) S-NR/CNTs-0.94 and (c) S-NR/CNTs-2.78 with a pre-set strain of $100 \%$.

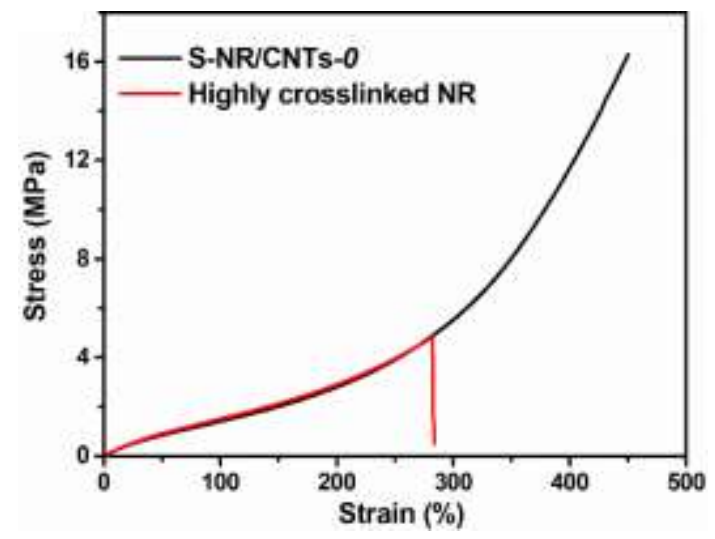

Figure S7. Representative stress-strain curves of S-NR/CNTs-0 and highly crosslinked NR. 

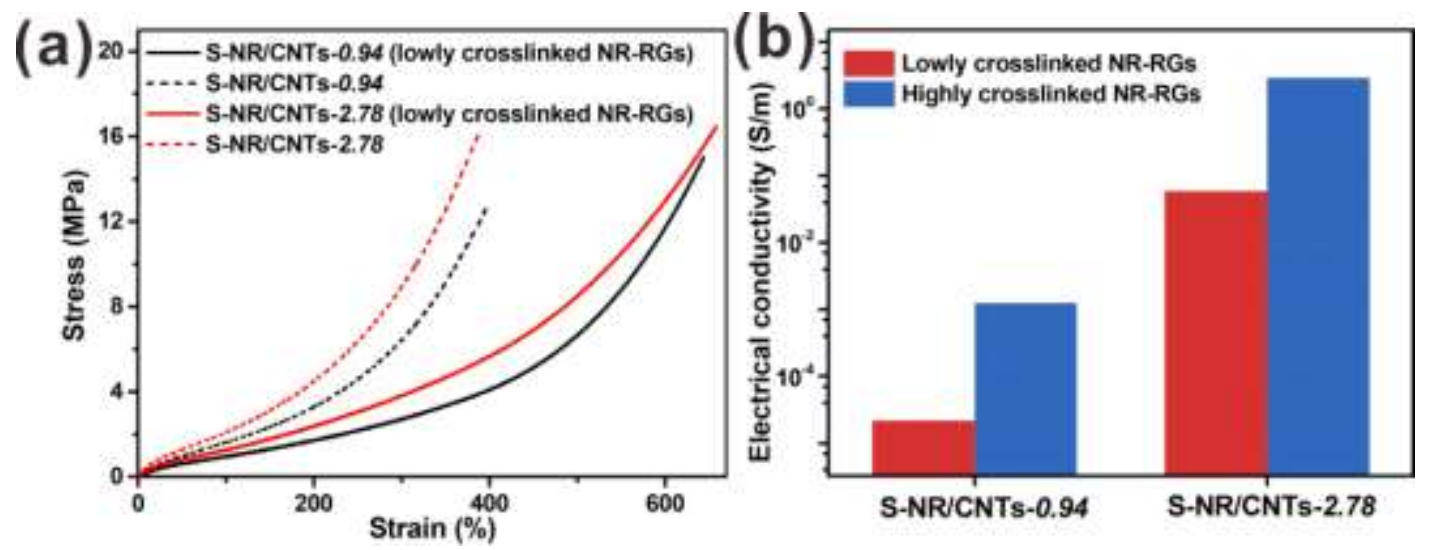

Figure S8. (a) Stress-strain curves and (b) electrical conductivity for S-NR/CNTs- $x$ based on highly and lowly crosslinked NR-RGs.

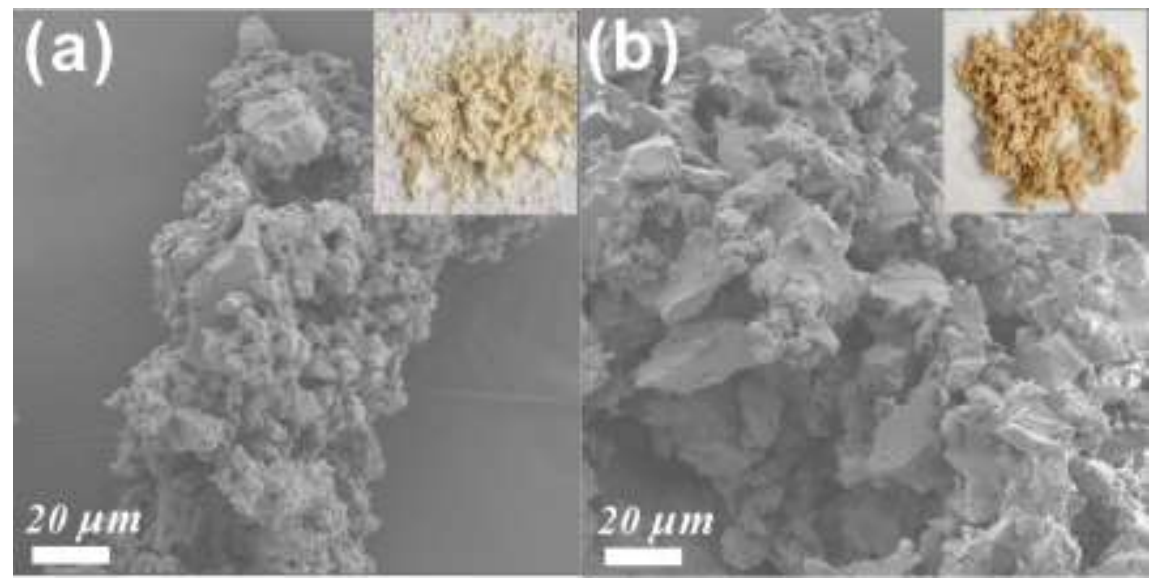

Figure S9. SEM images of (a) SBR-RGs and (b) NBR-RGs. Insets are the digital photographs of the corresponding rubber granules. 


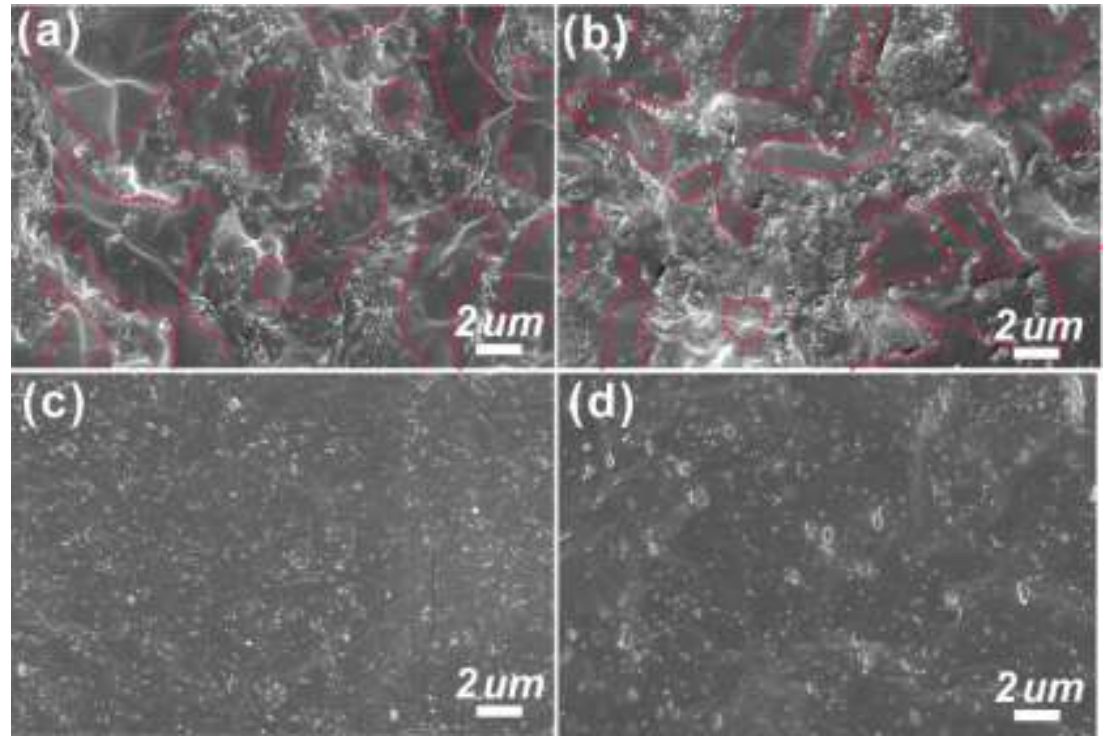

Figure S10. SEM images of (a) S-SBR/CNTs-4.54, (b) S-NBR/CNTs-4.54, (c) H-SBR/CNTs-4.54 and (d) HNBR/CNTs-4.54.
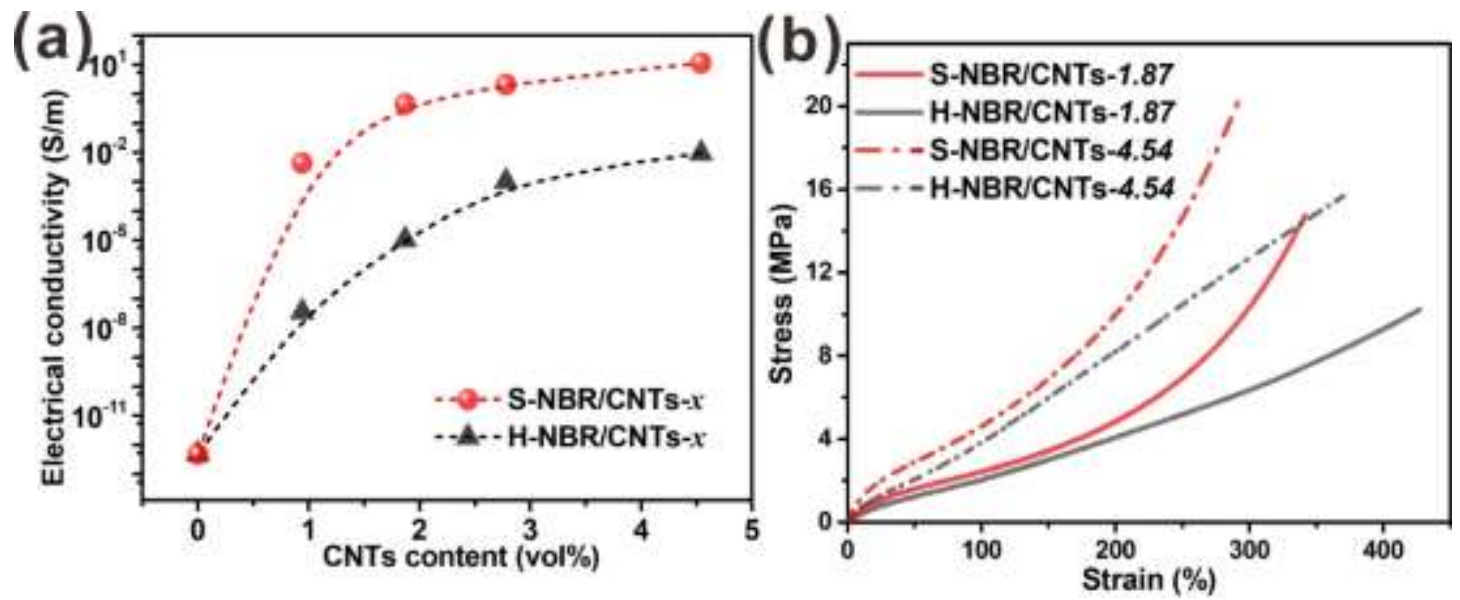

Figure S11. (a) Electrical conductivity and (b) representative stress-strain curves of segregated and homogeneous NBR/CNT composites. 

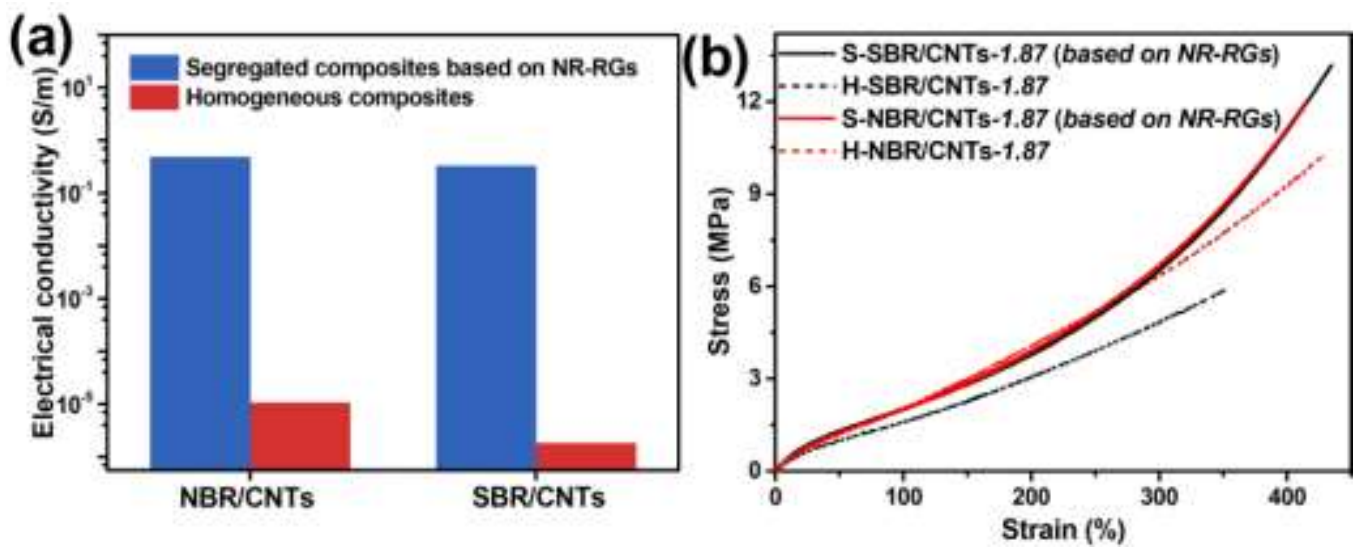

Figure S12. (a) Electrical conductivity and (b) representative stress-strain curves for S-SBR/CNTs-1.87 and S-

NBR/CNTs-1.87 composites based on NR-RGs and their homogeneous counterparts. 
Table S3. Crosslinking density and the fraction of specific sulfidic bridges in highly crosslinked NR and lowly crosslinked NR.

\begin{tabular}{ccccc}
\hline & $\begin{array}{c}\text { Total crosslinking } \\
\text { density } \\
\left(10^{-4} \mathrm{~mol} / \mathrm{cm}^{3}\right)\end{array}$ & $\begin{array}{c}\text { Monosulfidic } \\
\text { crosslinking density } \\
\left(10^{-4} \mathrm{~mol} / \mathrm{cm}^{3}\right)\end{array}$ & $\begin{array}{c}\text { Di- and polysulfidic } \\
\text { crosslinking density } \\
\left(10^{-4} \mathrm{~mol} / \mathrm{cm}^{3}\right)\end{array}$ & $\begin{array}{c}\text { Content of } \\
\text { di- and polysulfidic } \\
\text { crosslinking density }\end{array}$ \\
\hline $\begin{array}{c}\text { Lowly } \\
\text { crosslinked NR } \\
\text { Highly }\end{array}$ & 1.45 & 0.24 & 1.21 & $83.5 \%$ \\
crosslinked NR & 2.53 & 0.03 & 2.50 & $98.8 \%$ \\
\hline
\end{tabular}

Table S4. Comparison on the electrical conductivity and mechanical properties between segregated NR/CNT composites and previously reported CNT-filled rubber composites.

\begin{tabular}{lccccc}
\hline Rubber & CNT content & $\begin{array}{c}\text { Electrical } \\
\text { conductivity } \\
(\mathrm{S} / \mathrm{m})\end{array}$ & $\begin{array}{c}\text { Strength } \\
(\mathrm{MPa})\end{array}$ & $\begin{array}{c}\text { Tensile } \\
\text { modulus } \\
(\mathrm{MPa})\end{array}$ & Reference \\
\hline NR & $\begin{array}{c}0.94 \mathrm{vol} \% \\
(2 \mathrm{phr}) \\
2.78 \mathrm{vol} \% \\
(6 \mathrm{phr})\end{array}$ & $1.3 \times 10^{-3}$ & 12.4 & 6.8 & This work \\
& $5.4 \mathrm{wt} \%$ & 15.3 & 9.0 & \\
NR & $5 \mathrm{wt} \%$ & $\sim 1.4 \times 10^{-5}$ & $\sim 7.0$ & $\sim 2.5$ & 1 \\
NR & $5 \mathrm{phr}$ & $\sim 4.0 \times 10^{-5}$ & 15.1 & 7.7 & 2 \\
NR & $5 \mathrm{wt} \%$ & $2.0 \times 10^{-8}$ & 17.8 & $\sim 3.5$ & 3 \\
NR & $2 \mathrm{phr}$ & $\sim 3.3 \times 10^{-4}$ & $\sim 7.5$ & & \\
NR & $\sim 1.0 \times 10^{-5}$ & $\sim 7.7$ & $\sim 3.5$ & 5 \\
\hline
\end{tabular}




\begin{tabular}{|c|c|c|c|c|c|}
\hline NR & $3 \mathrm{phr}$ & $5.4 \times 10^{-4}$ & $\sim 17.0$ & $\sim 8.0$ & 6 \\
\hline ENR & $4 \mathrm{phr}$ & $\sim 2.0 \times 10^{-3}$ & $\sim 22.8$ & $\sim 3.8$ & 7 \\
\hline ENR & $5 \mathrm{phr}$ & $\sim 8.0 \times 10^{-6}$ & $\sim 7.5$ & $\sim 2.6$ & 8 \\
\hline NBR/ NR & $6 \mathrm{phr}$ & $\sim 0.09$ & $\sim 18.0$ & $\sim 8.8$ & 9 \\
\hline NBR & $4 \mathrm{phr}$ & 0.15 & 35.1 & $\longrightarrow$ & 10 \\
\hline NBR & $5 \mathrm{phr}$ & $\sim 2.0 \times 10^{-5}$ & $\sim 8.2$ & $\overline{ }$ & 11 \\
\hline $\begin{array}{l}\text { HXNBR/H } \\
\text { NBR }\end{array}$ & $5 \mathrm{phr}$ & $1.17 \times 10^{-5}$ & 15.2 & 3.23 & 12 \\
\hline SBR & $4 \mathrm{phr}$ & $\sim 5.0 \times 10^{-5}$ & 2.85 & 0.86 & 13 \\
\hline SBR & $4 \mathrm{phr}$ & $\sim 1.0 \times 10^{-7}$ & 3.4 & 2.32 & 14 \\
\hline SBR & $5 \mathrm{phr}$ & $\sim 1.0 \times 10^{-3}$ & 4.2 & $\sim 2.8$ & 15 \\
\hline SBR & $5 \mathrm{phr}$ & $4.9 \times 10^{-6}$ & $\sim 5.8$ & $\sim 4.6$ & 16 \\
\hline SEBS & $2.5 \mathrm{wt} \%$ & $\sim 0.5$ & $\sim 16.4$ & 6.8 & 17 \\
\hline SBR/BR & $5 \mathrm{phr}$ & $\sim 0.01$ & $\sim 4.3$ & 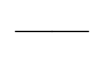 & 18 \\
\hline SBR/BR & $5 \mathrm{phr}$ & 0.95 & 4.05 & - & 19 \\
\hline EPDM & $3 \mathrm{phr}$ & $3.54 \times 10^{-5}$ & 4.5 & 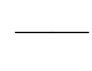 & 6 \\
\hline BR & $10 \mathrm{phr}$ & $1.8 \times 10^{-3}$ & $\sim 2.2$ & 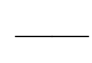 & 20 \\
\hline
\end{tabular}

Epoxide natural rubber (ENR), hydrogenated carboxylated nitrile rubber (HXNBR), hydrogenated nitrile rubber (HNBR), poly[styrene-b-(ethylene-co-butylene)-b-styrene] elastomer (SEBS), butadiene rubber (BR), ethylenepropylene-diene-monomer rubber (EPDM). 


\section{Reference}

(1) Bhattacharyya, S.; Sinturel, C.; Bahloul, O.; Saboungi, M.; Thomas, S.; Salvetat, J. Improving Reinforcement of Natural Rubber by Networking of Activated Carbon Nanotubes. Carbon 2008, 46, 10371045.

(2) Krainoi, A.; Nakaramontri, Y.; Wisunthorn, S.; Pichaiyut, S.; Nakason, C.; Kummerlöwe, C.; Vennemann, N.; Kiatkamjornwong, S. Influence of Carbon Nanotube and Ionic Liquid on Properties of Natural Rubber Nanocomposites. Express Polym. Lett. 2019, 13, 327-348.

(3) Abdullateef, A. A.; Thomas, S. P.; Al-Harthi, M. A.; De, S. K.; Bandyopadhyay, S.; Basfar, A. A.;

Atieh, M. A. Natural Rubber Nanocomposites with Functionalized Carbon Nanotubes: Mechanical, Dynamic Mechanical, and Morphology Studies. J. Appl. Polym. Sci. 2012, 125, E76-E84.

(4) Deng, F.; Ito, M.; Noguchi, T.; Wang, L.; Ueki, H.; Niihara, K.; Kim, Y. A.; Endo, M.; Zheng, Q. Elucidation of the Reinforcing Mechanism in Carbon Nanotube/Rubber Nanocomposites. ACS Nano 2011, $5,3858-3866$.

(5) Bokobza, L. Multiwall Carbon Nanotube-Filled Natural Rubber: Electrical and Mechanical Properties. Express Polym. Lett. 2012, 6, 213-223.

(6) Lorenz, H.; Fritzsche, J.; Das, A.; Stöckelhuber, K. W.; Jurk, R.; Heinrich, G.; Klüppel, M. Advanced Elastomer Nano-Composites Based on CNT-Hybrid Filler Systems. Compos. Sci. Technol. 2009, 69, 21352143.

(7) Krainoi, A.; Kummerlöwe, C.; Nakaramontri, Y.; Vennemann, N.; Pichaiyut, S.; Wisunthorn, S.; Nakason, C. Influence of Critical Carbon Nanotube Loading on Mechanical and Electrical Properties of Epoxidized Natural Rubber Nanocomposites. Polym. Test. 2018, 66, 122-136.

(8) Tang, Z.; Huang, Q.; Liu, Y.; Chen, Y.; Guo, B.; Zhang, L. Uniaxial Stretching-Induced Alignment of Carbon Nanotubes in Cross-Linked Elastomer Enabled by Dynamic Cross-Link Reshuffling. ACS Macro Lett. 2019, 8, 1575-1581. 
(9) Kueseng, P.; Sae-oui, P.; Rattanasom, N. Mechanical and Electrical Properties of Natural Rubber and Nitrile Rubber Blends Filled with Multi-Wall Carbon Nanotube: Effect of Preparation Methods. Polym. Test. 2013, 32, 731-738.

(10) Shao, C.; Wang, Q.; Mao, Y.; Li, Q.; Wu, C. Influence of Carbon Nanotubes Content on the Properties of Acrylonitrile-Butadiene Rubber/Cobalt Chloride Composites. Mater. Res. Express 2019, 6, 075323.

(11) Szadkowski, B.; Marzec, A.; Zaborski, M. Effect of Different Carbon Fillers on the Properties of Nitrile Rubber Composites. Compos. Interfaces 2019, 26, 729-750.

(12) Yin, Q.; Wen, Y.; Jia, H.; Hong, L.; Ji, Q.; Xu, Z. Enhanced Mechanical, Dielectric, Electrical and Thermal Conductive Properties of HXNBR/HNBR Blends Filled with Ionic Liquid-Modified Multiwalled Carbon Nanotubes. J. Mater. Sci. 2017, 52, 10814-10828.

(13) Bokobza, L. Multiwall Carbon Nanotube Elastomeric Composites: A Review. Polymer 2007, 48, 4907-4920.

(14) Bokobza, L.; Belin, C. Effect of Strain on the Properties of a Styrene-Butadiene Rubber Filled with Multiwall Carbon Nanotubes. J. Appl. Polym. Sci. 2007, 105, 2054-2061.

(15) Bokobza, L.; Rahmani, M.; Belin, C.; Bruneel, J.; El Bounia, N. Blends of Carbon Blacks and Multiwall Carbon Nanotubes as Reinforcing Fillers for Hydrocarbon Rubbers. J. Polym. Sci., Part B: Polym. Phys. 2008, 46, 1939-1951.

(16) Li, F.; Lu, Y.; Liu, L.; Zhang, L.; Dai, J.; Ma, J. Relations between Carbon Nanotubes' Length and Their Composites' Mechanical and Functional Performance. Polymer 2013, 54, 2158-2165.

(17) Li, Y.; Shimizu, H. Toward a Stretchable, Elastic, and Electrically Conductive Nanocomposite: Morphology and Properties of Poly[styrene-b-(ethylene-co-butylene)-b-styrene]/Multiwalled Carbon Nanotube Composites Fabricated by High-Shear Processing. Macromolecules 2009, 42, 2587-2593.

(18) Das, A.; Stöckelhuber, K. W.; Jurk, R.; Saphiannikova, M.; Fritzsche, J.; Lorenz, H.; Klüppel, M.; Heinrich, G. Modified and Unmodified Multiwalled Carbon Nanotubes in High Performance SolutionStyrene-Butadiene and Butadiene Rubber Blends. Polymer 2008, 49, 5276-5283. 
(19) Das, A.; Stöckelhuber, K. W.; Jurk, R.; Fritzsche, J.; Klüppel, M.; Heinrich, G. Coupling Activity of Ionic Liquids between Diene Elastomers and Multi-Walled Carbon Nanotubes. Carbon 2009, 47, 33133321.

(20) Hu, H.; Gao, Q.; Tian, G.; Hong, S.; Zhao, J.; Zhao, Y. The Influence of Topology and Morphology of Fillers on the Conductivity and Mechanical Properties of Rubber Composites. J. Polym. Res. 2018, 25, 87. 